\title{
Analysis of different HCV NS4B domains for the development of global consensus sequence
}

\author{
H. KHAN, S. MURAD*
}

Molecular Immunology Lab, Health Care Biotech Department, Atta-ur-Rahman School of Applied Biosciences, National University of Sciences and Technology, 44000 Islamabad, Pakistan

Received January 7, 2015; revised February 16, 2015; accepted August 12, 2015

\begin{abstract}
Summary. - The non-structural 4B (NS4B) protein of hepatitis C virus (HCV) is a hydrophobic protein implicated recently in the formation of membranous web, a platform for the formation of replication complex and thus is potential target for antivirals. The CLC main workbench was used to generate genotype-specific consensus sequence, global consensus sequence and a representative phylogenetic tree from non-structural $4 \mathrm{~B}$ (NS4B) protein sequences of seven different HCV genotypes reported from all over the world. The Cterminal domain (CTD) of NS4B protein especially the residues involved in interaction with ER membrane were found to be highly conserved. Other residues found to be highly conserved across all HCV genotypes included; 5 aromatic residues of N-terminal domain (NTD) (F49, W50, W55, F57, and Y63), 3 hydrophobic leucine residues (L237, L240, L245), and 2 positively charged residues of CTD (R248 and H250), dimerization motif of transmembrane domain 3 (TMD3) $\left(\mathrm{G}_{143} \mathrm{YGAG}_{147}\right)$ and its surrounding residues (F118 and F155) and TMD1 Ser/Thr cluster residues (T87, S88 and T95) involved in the hydrogen (H) bond interactions. In short, amino acids of NTD, TMD and CTD domains involved in the membrane association/anchoring of NS4B and formation of membranous web are highly conserved and can serve as potential targets for antivirals and peptide vaccines. These conserved residues formed the basis for the development of five short peptides proposed to serve as potential therapeutic target. The phylogenetic analysis was particularly interesting for NS4B sequences of 3a Pakistani isolates. The high degree of variability prevented the clustering of Pakistani isolates with other sequences in phylogenetic tree, revealing geographical disparity.
\end{abstract}

Keywords: HCV NS4B; global consensus sequence; membranous web; amphipathic alpha-helices; dimerization motif

\section{Introduction}

Hepatitis $\mathrm{C}$ is an enveloped, positive stranded RNA virus which accounts for 170 million chronic infections leading to hepatitis, fibrosis and even liver cancer (Han et al., 2011). Hepatitis C virus (HCV) belongs to the genus Hepacivirus of

*Corresponding author. E-mail: s.mall@asab.nust.edu.pk, sheebamall@yahoo.com; phone: +92-51-9085-6139.

Abbreviations: $\mathrm{CTD}=\mathrm{C}$-terminal domain; $\mathrm{HCV}=$ hepatitis $\mathrm{C}$ virus; $\mathrm{MB}=$ membranous web; $\mathrm{NS} 4 \mathrm{~B}=$ non-structural $4 \mathrm{~B} ; \mathrm{NTD}=\mathrm{N}$ terminal domain; $\mathrm{TMD}=$ transmembrane domain the family Flaviviridae (Miller et al., 1990; Simmonds et al., 2005). So far, there is no vaccination available for HCV. Due to high mutation rate, there are seven HCV genotypes showing $\sim 30 \%$ variability in their genetic sequences (Simmonds et al., 2005).The current standard therapies have genotype specific response rates. Most of HCV inhibitors are not designed to have pan-genotypic potential. The development of global consensus sequence against potential therapeutic targets involved in various stages of HCV replication may help to design the specific inhibitors with pan-genotypic effects. Such global consensus sequences have been designed against various $\mathrm{HCV}$ proteins such as the glycoproteins, structural and non-structural proteins. No such consensus 
has been established for non-structural protein-4B (NS4B). The HCV genotype-specific effects of NS4B inhibitors such as clemizole and anguizole has been reported. Therefore, the development of a global consensus sequence for NS4B would help to design drugs with a global impact. Therefore, the goal of the current study is to explore the conserved regions of HCV NS4B as a possible drug target.

The HCV viral genome is $9.6 \mathrm{~kb}$ long, encoding ten proteins in a single polypeptide chain which is later cleaved to give rise to ten proteins including; three structural proteins (core, E1, and E2), the highly hydrophobic p7 peptide, and six non-structural (NS) proteins (NS2, NS3, NS4A, NS4B, NS5A, and NS5B). The NS proteins are not only involved in $\mathrm{HCV}$ replication but some proteins such as; NS2, NS3, and NS5A also contribute to the viral assembly (Yi et al., 2007; Ma et al., 2008; Masaki et al., 2008; Tellinghuisen et al., 2008; Han et al., 2011). The NS3 acts as both serine protease and helicase (Kim et al., 1995; Tackett et al., 2001). The NS4A is a cofactor of NS3 and also helps in the binding of NS3 to the host membrane (Wolk et al., 2000). The NS5A binds to the HCV RNA and inhibits the action of interferon (Guo et al., 2001; Huang et al., 2005; Appel et al., 2008). The NS5B of $\mathrm{HCV}$ is RNA-dependent RNA polymerase which replicates $\mathrm{HCV}$ genome in association with other viral and host factors (Bartenschlager and Sparacio 2007; Moradpour et al., 2007; Stone et al., 2007).

The NS4B of HCV is a $27 \mathrm{~K}$ hydrophobic protein found to be an inducer of membranous web (Hugle et al., 2001; Moradpour et al., 2007). It comprises of an N-terminal domain (NTD) (aa 1 to 79), a transmembrane domain (TMD) harboring four putative transmembrane regions (aa 80 to 191), and a C-terminal domain (CTD) made from up of 70 amino acids (aa 192 to 261) (Lundin et al., 2003; Elazar et al., 2004; Aligo et al., 2009; Gouttenoire et al., 2009a,b). The $\mathrm{N}$-terminal part contains two alpha helices involved in NS4B host membrane association (Elazar et al., 2004; Gouttenoire et al., 2009a). The C-terminal domain (CTD) of NS4B consist of two putative $\alpha$-helices, represented by residues $\sim 201$ to 212 and $\sim 228$ to 253, respectively (Gouttenoire et al., $2009 \mathrm{~b}$ ). The CTD is believed to be on the cytosolic side of the endoplasmic reticulum membrane, whereas the location of the NTD remains controversial (Han et al., 2011). NS4B is a multi-functional protein that possesses GTPase and ATPase activities (Einav et al., 2004) and binds to the 3 ' end of the negative-sense viral RNA (Einav et al., 2008).

Like most of the positive sense RNA viruses, HCV also replicate their genome in association with the intracellular host membranes. These membranes are rearranged by the viruses in the form of novel structures termed as membranous web (Egger et al., 2002). Membranous webs have been observed in cells expressing mature NS4B suggesting that NS4B alone induces formation of the membranous webs (Egger et al., 2002; Konan et al., 2003). Membranous webs are named due to their appearance like membrane vesicles and are believed to be partly derived from the host endoplasmic reticulum. It provides a platform for the viral replication and harbors replication complex including the five replicase proteins (NS3, NS4A, NS4B, NS5A and NS5B) some viral RNAs and host factors (Aligo et al., 2009; Manna et al., 2010).

Owing to its multi-functionality and role in the induction of membranous webs, NS4B is a new target for the development of antivirals. There is a need to generate a global consensus sequence of NS4B across all HCV genotypes that can serve as potential target for the development of vaccines and antiviral therapies. This can be achieved by aligning available protein sequences of NS4B of different HCV genotypes. Aim of the current study is to develop a global consensus sequence of NS4B protein based on the individual consensus sequences of seven different HCV genotypes and the analysis of different domains of NS4B protein for variability or conservation. Finally, developing a phylogenetic tree would help in analyzing the evolutionary association of NS4B sequences of Pakistani isolates.

\section{Materials and Methods}

Retrieval of HCV NS4B sequences. The search for the protein sequences of HCV NS4B from the European HCV database and NCBI databases retrieved 701 sequences (Supplementary Table 1) belonging to seven genotypes and subtypes. These sequences were reported from all over the world including USA, UK, France, China, India, Pakistan, Turkey, South Korea, Australia, Taiwan, Japan, Brazil, Canada, Egypt, Spain, Denmark, Canada etc. 538 sequences were selected for different subtypes of genotype 1 that were reported from USA, China, Ireland, Australia, Denmark, Russia, Turkey, Japan, India, Indonesia and Equatorial Guinea. For different subtypes (2a, 2b, 2c, $2 \mathrm{i}$ and $2 \mathrm{k}$ ) of genotypes 2, 32 NS4B sequences that were reported from Japan, Vietnam, USA and Philippines were selected. Forty three sequences of NS4B for genotype 3 (subtypes 3a, 3g, 3i and $3 \mathrm{k}$ ) of HCV submitted from Pakistan, India, Japan, United Kingdom and Canada were included in the study. Thirty two sequences of NS4B of HCV genotype 4 from subtypes, $4 \mathrm{a}, 4 \mathrm{~b}$, $4 \mathrm{~d}, 4 \mathrm{f}, 4 \mathrm{~g}, 4 \mathrm{i}, 4 \mathrm{k}, 4 \mathrm{~m}, 4 \mathrm{n}, 4 \mathrm{o}, 4 \mathrm{p}, 4 \mathrm{q}, 4 \mathrm{r}$, and $4 \mathrm{v}$ were selected. These sequences were reported from United Kingdom, Canada, Egypt, USA and France. HCV genotype 5a sequences of NS4B reported from South Africa and China were retrieved. Forty five sequences of genotype 6 subtypes $6 \mathrm{a}, 6 \mathrm{~b}, 6 \mathrm{c}, 6 \mathrm{~d}, 6 \mathrm{e}, 6 \mathrm{f}, 6 \mathrm{~g}$, $6 \mathrm{~h}, 6 \mathrm{i}, 6 \mathrm{j}, 6 \mathrm{k}, 6 \mathrm{l}, 6 \mathrm{~m}, 6 \mathrm{n}, 6 \mathrm{o}, 6 \mathrm{p}, 6 \mathrm{q}, 6 \mathrm{t}$ with their origin from Thailand, Canada, Vietnam, China, Hong Kong and USA were used in the current study. A single available NS4B sequence of 7 a was used as a representative of genotype 7 .

Development of consensus sequence. The NS4B protein sequences of all six genotypes were fed to CLC main workbench software to 
generate consensus sequence of each genotype. The consensus sequences of six genotypes thus constructed in CLC main workbench software along with single 7 a sequence were subjected to alignment in CLC workbench to generate NS4B global consensus sequence of all seven genotypes.

Peptide designing and phylogenetic analysis. Different motifs and domains of NS4B were analyzed to find variation using HCV NS4B global consensus sequence. From HCV NS4B global consensus sequence alignment, short stretches of amino acids were selected from highly conserved region of HCV NS4B. These short peptides could be good targets for potential vaccine testing and development. A representative phylogenetic tree of NS4B protein sequences was constructed using UPGMA method.

\section{Results}

Protein consensus sequence of HCV NS4B was generated for each of the seven genotypes using CLC main workbench (Fig. 1). Global consensus sequence of 701 protein sequences of NS4B was developed by aligning the consensus sequences of all six genotypes and a 7a sequence (Fig. 1). The bars represent percent conservation of amino acid residues. The " $\mathrm{X}$ " shows highly variable residues whereas conserved amino acid residues are labeled by their symbols. Different regions of global consensus sequences were analyzed for amino acid variability and conservation. Stretches of highly conserved amino acid residues can serve as peptide vaccine. Therefore, short peptides as shown in Table 1 and highlighted in consensus sequence (Fig. 1) were selected from the conserved regions of NS4B. Phylogenetic analysis was done by aligning first all 701 NS4B sequences in CLC workbench followed by drawing phylogenetic tree using UPGMA method. From the phylogenetic tree of 701 NS4B sequences, 210 NS4B representative sequences of different HCV genotypes were selected for the construction of ultimate phylogenetic tree (Supplementary Fig. 1). The excluded NS4B sequences included sequences of same HCV subtype which clustered together and did not show any evolutionary association with NS4B sequences of other HCV genotypes and subtypes. Five NS4B sequences of 3a Pakistani isolates were also aligned in order to analyze the variability and conservation of various residues among them (Fig. 2).

Table 1. Sequences and positions of peptides designed from the highly conserved regions of NS4B

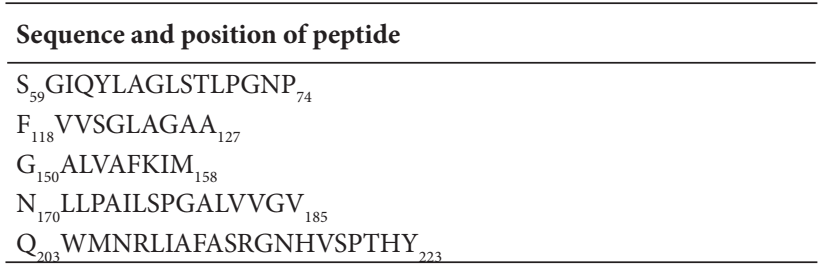

\section{Discussion}

Little is known about the functions of ER-anchored NS4B and phosphorylated form of NS5A, however both are critical players in the genome replication of HCV (Blight, 2011). The NS4B is anchored in the ER membrane through its four transmembrane domains whereas, the $\mathrm{N}$ and $\mathrm{C}$-terminal domains are presumably localized towards the cytosolic side of ER membrane (Blight, 2011). The NS4B protein is comprised of three distinct regions; an N-terminal domain (NTD), transmembrane domain (TMD) and a C-terminal domain (CTD) (Lundin et al., 2003; Elazar et al., 2004; Aligo et al., 2009; Gouttenoire et al., 2009a,b).

The NTD is 1-79 aa long and contains two amphipathic alpha-helices (AH1 and AH2) (Elazar et al., 2004; Gouttenoire et al., 2009a). The AH1 (encompassing 6-29 aa) is presumed to interact with ER membrane through its hydrophobic side. Disrupting the helical structure of AH1 is associated with the loss of NS4B ability to form membranous web and replication complex (Elazar et al., 2004). The AH2 (42-66 aa long) also mediates ER membrane interaction of NS4B and is critical for the oligomerization of NS4B (Lundin et al., 2003; Elazar et al., 2004). The global consensus sequence analysis shows that $\mathrm{N}$ terminal domain is comparatively more variable. However, the second $\mathrm{AH} 2$ is relatively more conserved highlighting its role in the ER membrane association and oligomerization of NS4B. The last portion of NTD (59-74 aa) has been found to be well conserved among different $\mathrm{HCV}$ genotypes. Most of the mutated residues in $\mathrm{AH} 2$ and in the last part of NTD exhibit similar physiochemical properties with the wild type which has been reported earlier (Gouttenoire et al., 2009a). Moreover, the replacement of the aromatic residues W50, W55, F57, and Y63 of AH2, is believed to play an essential role in ER membrane interface interaction, and together with alanine (A) abrogates formation of membranous web and viral replication (Gouttenoire et al., 2009a). These residues were found to have high degree of conservation across different HCV genotypes.

The central region of NS4B consists of four transmembrane domains (TMD1 to TMD4). Besides its functions as ER membrane anchoring of NS4B, the transmembrane domains are implicated in recruitment of viral and host proteins to the replication complex and the formation of NS4B foci/membranous web. The TMD2 and TMD3 contain dimerization motif GlyXXXGly (GXXXG), a common feature of TMD helices (Han et al., 2011). Mutation of glycine in dimerization motif such as G125A/L and G129L is associated with in vitro suppression of $\mathrm{HCV}$ replication (Han et al., 2011). Our global consensus sequence shows that all amino acid residues of TMD2 dimerization motif $\left(G_{125} A A V G_{129}\right)$ are highly conserved except V128I substitution in genotype 1 and 3 . Among the surrounding residues (F118 and V128) of TMD2 dimerization contributing to 


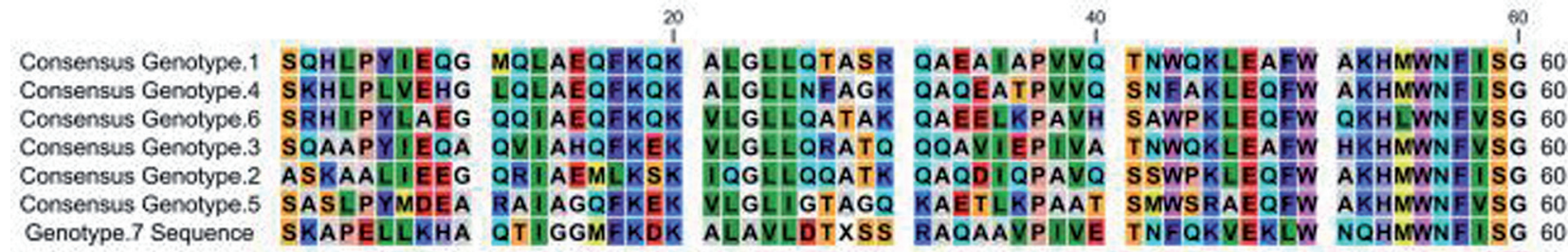
Consensus SXHLPYIEEG QQIAEQFKQK XLGLLQTAXK QAXXIKPAVQ SNWQKLEQFW AKHMWNFISG Conservation $\mathrm{sm}$ 100 Consensus Genotype.1 IQYYLAGESTL PGNPAIASEM AFTAAVTSPE TTQQTLEFNII EGGWVAAQLA APGAATAFVG 120 Consensus Genotype.4 PQYLAGLSTL PGNPAIASLM SFTAAVTSPL TTQQTLLENI EGGWVASQIA TPTASTAFVV 120 Consensus Genotype.6 IQYLAGLSTL PGNPAVASLM SFSASLTSPL STSTTLLLNI LGGWVASQLA PPTASTAFVN 120 Consensus Genotype. 3 IQYLAGLSTL PGNPAVASLM AFTASVTSPL TTNQTMFFNI LGGWVATHLA GPQSSSAFVV 120 Consensus Genotype.2 IOYLAGLSTL PGNPAVASMM AFSAALTSPL STSTTILLNI MGGWLASQIA PPAGATGFVV 120 Consensus Genotype.5 IQYLAGLSTL PGNPAVATEM SFTAAVTSPL TTQQTLLFNI EGGWVASQIA PPTAATAFVV 120 Genotype.7 Sequence IQYELAGLSTL PGNPTVASLM AFTASVTSPL ATSTTLLVNI EGGWEASQLA PPSAATTEVV 120 Consensus IOYLAGLSTL PGNPAVASLM AFTAAVTSPL TTXQTLLFNI LGGWVASQLA PPTAATAFVV

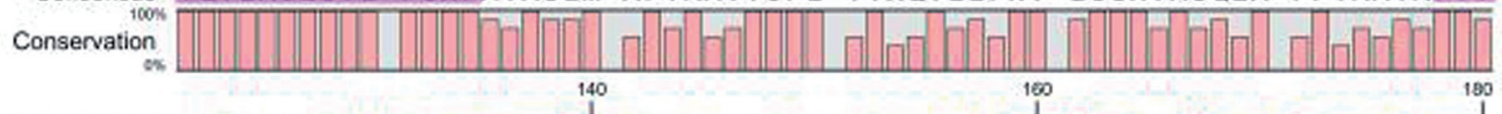

Consensus Genotype.1 AGLAGAAIGS VGEGGELVDI LAG YYGAGVAG ALVAFKIMSG EVPSTEDLVN ILPAILSPGA 180 Consensus Genotype.4 SGLAGAAVGS VGLGKILVDI LAG GAGVAG AVVTFKIIMSG EMPSTEDLVN LIPAILSPGA 180 Consensus Genotype.6 SGLAGAAVGS FGEGRYLVDI LAGYGAGVSG ALVAFKIMSG EXPSTEDMVN LLPALLSPGA 180 Consensus Genotype.3 SGLAGAA GG IGLGRLLDI LAG YGAGVG ALVAEK IMGG ELPTAEDMVN LLPAILSPGA 180 Consensus Genotype.2 SGLVGAAVGS IGLGKILVDX LAG YGAGISG ALVAFKIMSG EKPSVEDVVN LLPAILSPGA 180 Consensus Genotype.5 SGMAGAAVGS IGLGRVLIDI LAG YGAGVAG ALVAFKIMCG EKPXAEDLVN LLPSILCPGA 180 Genotype.7 Sequence SGLAGAAVGS VGLGEVLVDV LAGMAGIAG ALVAEKIMSG EVPSTEDLAN LLPAILSPGA 180 Consensus SGLAGAAVGS IGLGKVLVDI LAGYGAGVAG ALVAFKIMSG EXPSTEDLVN LLPAILSPGA

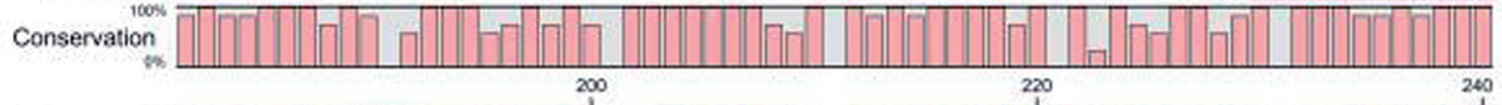
Consensus Genotype.1 LVVGVVCAAI LRRHVGPGEG AVQWMNRLIA FASRGNHVSP THYVPESDAA ARVTQILSSL 240
Consensus Genotype.4 LVVGVVCAAI LRRHVGPGEG AVQWMNRLIA FASRGNHVSP THYVPESDAA ARVTQILSSL 240 Consensus Genotype.4 LVVGVVCAAI LRRHVGPGEG AVQWMNRLIA FASRGNHVSP THYVPESDAA ARVTQILSSL 240
Consensus Genotype.6 LVVGVVCAAI LRRHVGPAEG ANQWMNRLIA FASRGNHVSP THYVPETDAS RNVTQILSSL 240 Consensus Genotype.3 LVVGVICAAI LRRHVGPGEG AVQWMNRLIA FASRGNHVSP THYVPESDAA ARVTALLSSL 240 Consensus Genotype.2 LVVGVICAAII LRRHVGQGEG AVQWMNRLIA FASRGNHVAP THYVTESDAS QRVTQLLSSL 240 Consensus Genotype.5 LVVGVICAAV LRRHIGPGEG AVQWMNRLI A FASRGNHVSP THYVPETDAS AKVTQELSSL 240 Genotype.7 Sequence LVVGVVCAAI IKRHTGTSEG VTQWMNRLIA EASBGNHVSP THYIQDDDAS KRVMGILSSL 240 Consensus, LVVGVVCAA I LRRHVGPGEG AVQWMNRLIA FASRGNHVSP THYVPESDAS ARVTQILSSL
Conservation
of 260

Consensus Genotype.1 TVTQLLRREH QWTSSDCTTP C 261 Consensus Genotype.4 TVTSLLRRLH KWINEDCSTP C 261 Consensus Genotype.6 TITSLLRRLH EWINEDWATP C 261 Consensus Genotype.3 TVTSLLRRLH QWI NEDYPSP C 261 Consensus Genotype.2 TITSLLRREH NWITEDCPVP C 261 Consensus Genotype.5 TVTSLLKRLH TWIGEDYSTP C 261 Genotype.7 Sequence TITSLIKRVL AWAQTXYSAP C 261 Consensus TVTSLLRRLH QWINEDXSTP C Conservation

Fig. 1

Multiple sequence alignment of NS4B protein consensus sequences of genotypes 1-6 and a single available NS4B protein sequence of genotype 7a The global consensus sequence is shown at the base. The conserved residues are represented by their symbols while "X" denotes highly variable residues. Bars under the global consensus sequence show percent degree of amino acid conservation. Stretches of amino acids highlighted in light purple in the global consensus sequence show short peptides. 
ADR73013.1 SQAAPYIEQA QVIAHQFKEK VLGLLQRATQ QPAVIEPIVV ACT79637.1 SQAAPYIEQA QVIAHQFKEK VLGLLQRATQ QQAVIEPIVV T ADI58378.1 SQAAPYIEQA QVIAHQFKEK VLGLLQRATQ QQAVIEPIVN AED02428.1 SQAAPYIEQA QVIAHQFKEK VLGLLQRATQ QQAVIEPIVA AEO87022.1 SQAAPYIEQA QVIIAHQEKE

consensus SQAAPYIEQA QVIAHQFKEK VLGLLQRATQ QQAVIEPIVV Conservation

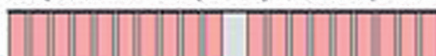
of $\frac{111010}{80}$ TNWQKLEA FW HKHMWNFVSG 60 TNWQKLETFW HKHMWNFIVSG 60 TNWQKLEAFW HKHMWNFVSG 60 TNWQKLGAFW HKHMGNFGSG 60

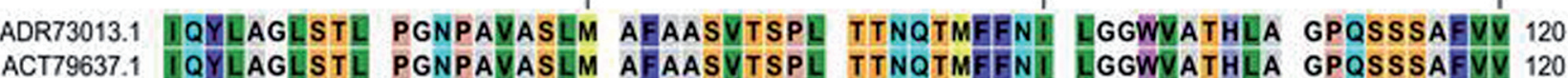
ADI58378.1 IQYLAGLSTL PGNPAVASLM AFTASVTSPL TANQTMFFNI LGGWVATHLA GPQSSSAFVV 120 AED02428.1 IQYLAGLSTL PDNPAVASLM AFTASVTSPL TTNQTMFFNII LGGWVATHLA GPQSSSAFVV 120 AED87022.1 IQYLTGLSTL PGNPAGASLM AFTASITSPI NTKQTMFFNI LGGWVATHLG GPQNFSPEVV 120 Consensus IQYLAGLSTL PGNPAVASLM AFTASVTSPL TTNQTMFFNI LGGWVATHLA GPQSSSAFVV Conservation $\sin ^{1000}$

140 160

I 180 ADR73013.1 SGLAGAAIGD IGLGRVLLEI LARYGAGFSG ALGAFKIMGG ELP...... N AEDVVNLLPA 174 ACT79637.1 SGLAGAAIGG IGLGRVLLDI LAGYGAGVSG ALVAFKIMGG ELP..... N AEDVVNLLPA 174 ADI58378.1 SGLAGAAIGG IGLGRVLHDI LAGYGAGVSG ALVAFKIMGG ELP..... T AEDMVNLLPA 174 AED02428.1 SGLAGAAIGG IGLGRVLLDI LAGYGAGVSG ALVAFKIMGG ELP...... A AEDVVNLLPA 174 AED87022.1 NRLARAAIIGG ISLSRVLLDII LARNGAGVSG ALVAFKNEGG GTPPWRERGQ SGARHNISGA 180 Consensus SGLAGAAIGG IGLGRVLLDI LAGYGAGVSG ALVAFKIMGG ELP...- N AEDVVNLLPA Conservation

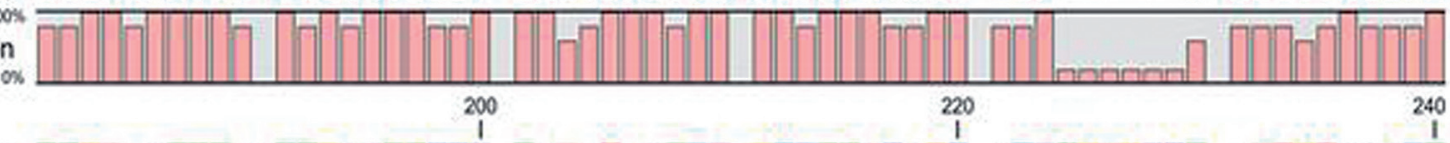

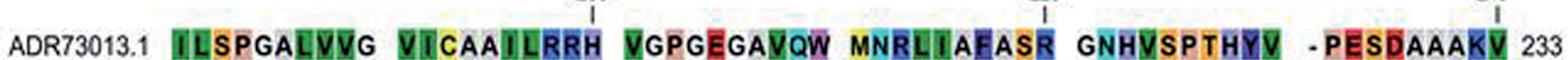
ACT79637.1 ILSPGALVVG VIICAALLRRH V̈GPGEGVQW MNRLIAAASR GNHVSPTHYV -PESDAAARV 233 ADI58378.1 ILSPGALVVG VIICAAILRRH VGGPGEGEVW MNRLIAAESR GNHVSPTHYV -PESDAAARV 233 AED02428.1 ILSPGALVVG VIICAAILRRH NGPGGGSSTM DEQAHCVRIS GQPRFTNALC -SRERRCGEG 233 AED87022.1 SPRRGDMW-. - - CHTTSAR - -GTRGGSIOW KNGFLR-SES GQPLETKHLIC SPRDDAAQKV 233 Consensus ILSPGALVVG VICAAILRRH VGPGEGAVQW MNRLIAFASR GNHVSPTHYV -PESDAAAXV

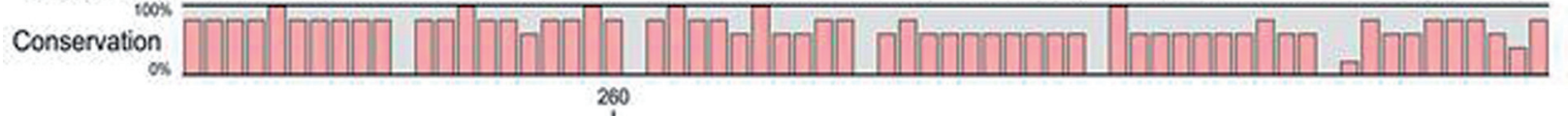

ADR73013.1 TALLSSLTD. TSLLRRLRQW INEDYPSPC 261 ACT79637.1 TALLSSETV. TSLLRRLHQW INEDYPSPC 261 ADI58378.1 TLLLLNSLTI. TTLLRRLHKW INEDYPSPC 261 AED02428.1 HALLIISINCH QSAPGDMISG INEDYPSS - 261 AED87022.1 TALAELLTV. TSLLGGYISG IKKNYPAPC 261 Consensus TALLSSLTV. TSLLRRLXXW INEDYPSPC Conservation (1)<smiles>C1=CC2CC12</smiles>

Fig. 2 under the consensus sequence show percent degree of amino acid conservation. 
hydrogen bond interaction (Adamian et al., 2001; Langosch et al., 2009), F118 was found to be highly conserved across all genotypes. However, V128I substitution might support the fact that V128A mutation has negligible effect on the Japanese fulminant hepatitis 1 replication as described previously (Han et al., 2011). The dimerization motif $\mathrm{G}_{143} \mathrm{YGAG}_{147}$ of TMD3 was found to be $100 \%$ conserved among all HCV genotypes. The closest F155 residue is also well conserved suggesting its importance in the hydrogen (H) bond interactions in TMD3.

The Ser/Thr (S/T) cluster-like dimerization motif of TMD1 promotes helical interaction through hydrogen bond of hydroxyl group in the side chain of Ser and/or Thr (Dawson et al., 2002; Langosch et al., 2009). The NS4B consensus sequence alignment reveals that some amino acid residues T87, S88, and T95 of Ser/Thr clusters required for the HCV replication (Han et al., 2011) are well conserved. However, T94Q was found be the most prevalent among the serine and threonine residues of Ser/Thr (S/T) cluster-like dimerization motif. The S83A mutation that suppresses HCV replication in vitro (Han et al., 2011) was not found; however some $\mathrm{HCV}$ genotypes harbor S83T substitution.

The C-terminal domain (CTD) of NS4B consist of two putative $a$-helices, represented by residues $\sim 201$ to 212 and $\sim 228$ to 253, respectively (Gouttenoire et al., 2009 b). The consensus sequence analysis shows that CTD has comparatively high degree of conservation. The hydrophobic leucine (L) residue at positions 237, 240, 246, and 249 has been implicated in an ER membrane interaction of NS4B (Gouttenoire et al., 2009 b), protein-protein interaction (Yu et al., 2006) or protein-RNA interactions (Egger et al., 2002) and is required for viral replication of HCV. Mutation of these leucines $(\mathrm{L})$ to alanine $(\mathrm{A})$ impairs membrane association of NS4B and replication of HCV (Gouttenoire et al., 2009b). Our NS4B consensus sequence analysis reveals that L237, L240, and L245 are highly conserved. However, L246I and L249V substitution were found in the NS4B of genotype 7. Both isoleucine and valine like leucine are hydrophobic in nature supporting the fact that these are involved in the vital activities associated with NS4B. The positively charged aa residues K247, R248, and H250 in CTD interact with the negative head groups of lipids of ER membrane and are required for HCV replication (Liefhebber et al., 2009). Substitution of these positively charged aa with a negatively charged glutamic acid (K247E/R248E/H250E) severely affect membrane association of NS4B and hence replication of HCV (Liefhebber et al., 2009). The consensus sequence analysis shows that these positively charged residues are highly conserved but genotype 7 carries H250L substitution. Yu et al. suggested that membrane association of NS4B is mediated by palmitoylation on cysteines 257 and 261 (Yu et al., 2006). Our consensus sequence analysis illustrates that residue at 257 is highly variable and rep- resented by " $\mathrm{X}$ " in global consensus sequence. However, C261 is well conserved across all HCV genotypes. It has been determined that C257S and C261S substitutions do not influence localization of the NS4B-CTD (Liefhebber et al., 2009). This might be an explanation for the variability of cysteine 257 .

In the middle of NS4B, there are two nucleotide binding motifs (NBMs), A motif and B motif characterized by conserved elements GXXXXGK and DXXA, respectively (Gorbalenya and Koonin, 1989; Sklan et al., 2006). It has been shown that mutations of NBM motifs residues impair GTP binding and hydrolysis ability of NS4B as well as inhibit viral replication (Sklan et al., 2006). This suggests that NBMs bind to RNA during HCV replication. The consensus sequence analysis of NS4B elucidates that residue G129 and G134 of motif A $\left(\mathrm{G}_{129}\right.$ SIGLGK $\left._{135}\right)$ and D228 of motif B $\left(\mathrm{D}_{228} \mathrm{ASA}_{231}\right)$ are highly conserved, however, K135 was found to be mutated with a similar positively charged residue, arginine in genotypes 3, 5 and 6. The A231 of motif B was found substituted by three different residues, $\mathrm{Q}, \mathrm{R}$, and $\mathrm{K}$ in genotypes 3, 6 and 7, respectively.

During the NS4B consensus sequence alignment a rooted phylogenetic tree of 210 NS4B representative sequences was constructed using UPGMA method. The phylogenetic analysis of NS4B shows that root of the tree bifurcated in to two branches, one leads to evolution of $3 \mathrm{a}$ (AED87022.1/Pak), second branch bifurcates further and from one wing arises $7 \mathrm{a}$ (EF108306) and from second wing evolved genotypes 1, 2, 3, 4, 5, 6 and their subtypes except 3a (AED87022.1/Pak). The phylogenetic analysis of NS4B shows that NS4B sequences of 3a Pakistani isolates do not clustered together and each has an evolutionary association with NS4B sequences of genotype 3 isolates reported from different countries. The phylogenetic analysis suggests that NS4B sequences of $3 \mathrm{a}$ isolates from Pakistan have high degree of sequence variability and hence are evolutionary distant from each other.

Our consensus sequence analysis of HCV NS4B suggests that there are certain stretches of amino acids in NTD, TMD and CTD which are involved in membrane association, anchoring, formation of replication complex and viral replication are highly conserved. These stretches of conserved residues can be used for the testing and development of peptide vaccine. Furthermore information about the conserved regions of NS4B could be very helpful for the development of antiviral compounds. The phylogenetic analysis and sequence alignment of NS4B suggests that there is high degree of sequence variability among the NS4B sequences of different $3 a$ isolates reported from Pakistan and evolutionary are not clustered together in the tree.

Supplementary information is available in the online version of the paper. 
Acknowledgements. We are obliged to National University of Sciences and Technology (NUST) for providing all kind of help and support.

\section{References}

Adamian L, Liang J (2001): Helix-helix packing and interfacial pairwise interactions of residues in membrane proteins. J. Mol. Biol. 311, 891-907. http://dx.doi.org/10.1006/ jmbi.2001.4908

Aligo J, Jia S, Manna D, Konan K V (2009): Formation and function of hepatitis $\mathrm{C}$ virus replication complexes require residues in the carboxy-terminal domain of NS4B protein. Virol. 393, 68-83. http://dx.doi.org/10.1016/j. virol.2009.07.033

Appel N. Zayas M, Miller S, Krijnse-Locker J, Schaller T, Friebe P, Kallis S, Engel U, Bartenschlager R (2008): Essential role of domain III of nonstructural protein $5 \mathrm{~A}$ for hepatitis $\mathrm{C}$ virus infectious particle assembly. PLoS. Pathog. 4, e1000035. http://dx.doi.org/10.1371/journal. ppat. 1000035

Bartenschlager R, Sparacio S (2007): Hepatitis C virus molecular clones and their replication capacity in vivo and in cell culture. Virus. Res. 127, 195-207. http://dx.doi. org/10.1016/j.virusres.2007.02.022

Blight KJ (2011): Charged residues in hepatitis C virus NS4B are critical for multiple NS4B functions in RNA replication. J. Virol. 85, 8158-8171. http://dx.doi.org/10.1128/ JVI.00858-11

Dawson JP, Weinger JS, Engelman DM (2002): Motifs of serine and threonine can drive association of transmembrane helices. J. Mol. Biol. 316, 799-805. http://dx.doi.org/10.1006/ jmbi.2001.5353

Egger D, Wolk B, Gosert R, Bianchi L, Blum HE, Moradpour D, Bienz K (2002): Expression of hepatitis $C$ virus proteins induces distinct membrane alterations including a candidate viral replication complex. J. Virol. 76, 5974-5984. http://dx.doi.org/10.1128/JVI.76.12.5974-5984.2002

Einav S, Elazar M, Danieli T, Glenn JS (2004): A nucleotide binding motif in hepatitis C virus (HCV) NS4B mediates HCV RNA replication. J. Virol. 78, 11288-11295. http://dx.doi. org/10.1128/JVI.78.20.11288-11295.2004

Einav S, Gerber D, Bryson PD, Sklan EH, Elazar M, Maerkl SJ, Glenn JS, Quake SR (2008): Discovery of a hepatitis C target and its pharmacological inhibitors by microfluidic affinity analysis. Nat. Biotechnol. 26, 1019-1027. http:// dx.doi.org/10.1038/nbt.1490

Elazar M, Liu P, Rice CM, Glenn JS (2004): An N-terminal amphipathic helix in hepatitis C virus (HCV) NS4B mediates membrane association, correct localization of replication complex proteins, and HCV RNA replication. J. Virol. 78, 11393-11400. http://dx.doi.org/10.1128/JVI.78.20.1139311400.2004

Gorbalenya AE, Koonin EV (1989): Viral proteins containing the purine NTP-binding sequence pattern. Nucleic.
Acids. Res. 17, 8413-8440. http://dx.doi.org/10.1093/ nar/17.21.8413

Gouttenoire J, Castet V, Montserret R, Arora N, Raussens V, Ruysschaert JM, Diesis E, Blum HE, Penin F, Moradpour D (2009a): Identification of a novel determinant for membrane association in hepatitis $\mathrm{C}$ virus nonstructural protein 4B. J. Virol. 83, 6257-6268. http://dx.doi. org/10.1128/JVI.02663-08

Gouttenoire J, Montserret R, Kennel A, Penin F, Moradpour D (2009b): An amphipathic alpha-helix at the $\mathrm{C}$ terminus of hepatitis $\mathrm{C}$ virus nonstructural protein $4 \mathrm{~B}$ mediates membrane association. J. Virol. 83, 11378-11384. http:// dx.doi.org/10.1128/JVI.01122-09

Guo JT, Bichko VV, Seeger C (2001): Effect of alpha interferon on the hepatitis C virus replicon. J. Virol. 75, 8516-8523. http://dx.doi.org/10.1128/JVI.75.18.8516-8523.2001

Han Q, Aligo J, Manna D, Belton K, Chintapalli SV, Hong Y, Patterson RL, van Rossum DB, Konan KV (2011): Conserved GXXXG- and S/T-like motifs in the transmembrane domains of NS4B protein are required for hepatitis C virus replication. J. Virol. 85, 6464-6479. http://dx.doi. org/10.1128/JVI.02298-10

Huang L, Hwang J, Sharma SD, Hargittai MR, Chen Y, Arnold JJ, Raney KD, Cameron CE (2005): Hepatitis C virus nonstructural protein $5 \mathrm{~A}$ (NS5A) is an RNA-binding protein. J. Biol. Chem. 280, 36417-36428. http://dx.doi. org/10.1074/jbc.M508175200

Hugle T, Fehrmann F, Bieck E, Kohara M, Krausslich HG, Rice CM, Blum HE, Moradpour D (2001): The hepatitis C virus nonstructural protein $4 \mathrm{~B}$ is an integral endoplasmic reticulum membrane protein. Virol. 284, 70-81. http:// dx.doi.org/10.1006/viro.2001.0873

Kim DW, Gwack Y, Han JH, Choe J (1995): C-terminal domain of the hepatitis $\mathrm{C}$ virus NS3 protein contains an RNA helicase activity. Biochem. Biophys. Res. Commun. 215, 160-166. http://dx.doi.org/10.1006/bbrc.1995.2447

Konan KV, Giddings TH, Ikeda M, Li K, Lemon SM, Kirkegaard K (2003): Nonstructural protein precursor NS4A/B from hepatitis $\mathrm{C}$ virus alters function and ultrastructure of host secretory apparatus. J. Virol. 77, 7843-7855. http://dx.doi. org/10.1128/JVI.77.14.7843-7855.2003

Langosch D, Arkin IT (2009): Interaction and conformational dynamics of membrane-spanning protein helices. Protein. Sci. 18, 1343-1358. http://dx.doi.org/10.1002/pro.154

Liefhebber JM, Brandt BW, Broer R, Spaan WJ, van Leeuwen HC (2009): Hepatitis C virus NS4B carboxy terminal domain is a membrane binding domain. J. Virol. 6, 62. http:// dx.doi.org/10.1186/1743-422X-6-62

Lundin M, Monne M, Widell A, Von Heijne G, Persson MA (2003): Topology of the membrane-associated hepatitis $\mathrm{C}$ virus protein NS4B. J. Virol. 77, 5428-5438. http://dx.doi. org/10.1128/JVI.77.9.5428-5438.2003

Ma Y, Yates J, Liang Y, Lemon SM, Yi M (2008): NS3 helicase domains involved in infectious intracellular hepatitis $\mathrm{C}$ virus particle assembly. J. Virol. 82, 7624-7639. http://dx.doi. org/10.1128/JVI.00724-08 
Manna D, Aligo J, Xu C, Park WS, Koc H, Heo WD, Konan KV (2010): Endocytic Rab proteins are required for hepatitis C virus replication complex formation. Virol. 398, 21-37. http://dx.doi.org/10.1016/j.virol.2009.11.034

Masaki T, Suzuki R, Murakami K, Aizak, H, Ishii K, Murayama A, Date T, Matsuura Y, Miyamura T, Wakita T, Suzuki $\mathrm{T}$ (2008): Interaction of hepatitis C virus nonstructural protein $5 \mathrm{~A}$ with core protein is critical for the production of infectious virus particles. J. Virol. 82, 7964-7976. http:// dx.doi.org/10.1128/JVI.00826-08

Miller RH, Purcell RH (1990): Hepatitis C virus shares amino acid sequence similarity with pestiviruses and flaviviruses as well as members of two plant virus supergroups. Proc. Natl. Acad. Sci. USA 87, 2057-2061. http://dx.doi. org/10.1073/pnas.87.6.2057

Moradpour D, Penin F, Rice CM (2007): Replication of hepatitis C virus. Nat. Rev. Microbiol. 5, 453-463. http://dx.doi. org/10.1038/nrmicro1645

Simmonds P, Bukh J, Combet C, Deleage G, Enomoto N, Feinstone S, Halfon P, Inchauspe G, Kuiken C, Maertens G, Mizokami M, Murphy DG, Okamoto H, Pawlotsky JM, Penin F, Sablon E, Shin IT, Stuyver LJ, Thiel HJ, Viazov S, Weiner AJ, Widell A (2005): Consensus proposals for a unified system of nomenclature of hepatitis $\mathrm{C}$ virus genotypes. Hepatol. 42, 962-973. http://dx.doi.org/10.1002/hep.20819

Sklan EH, Glenn JS (2006): HCV NS4B: From Obscurity to Central Stage.
Stone M, Jia S, Heo WD, Meyer T, Konan KV (2007): Participation of rab5, an early endosome protein, in hepatitis $\mathrm{C}$ virus RNA replication machinery. J. Virol. 81, 4551-4563. http://dx.doi.org/10.1128/JVI.01366-06

Tackett AJ, Wei L, Cameron CE, Raney KD (2001): Unwinding of nucleic acids by HCV NS3 helicase is sensitive to the structure of the duplex. Nucleic. Acids. Res. 29, 565-572. http://dx.doi.org/10.1093/nar/29.2.565

Tellinghuisen TL, Foss KL, Treadaway J (2008): Regulation of hepatitis $C$ virion production via phosphorylation of the NS5A protein. PLoS. Pathog. 4, e1000032. http://dx.doi. org/10.1371/journal.ppat.1000032

Wolk B, Sansonno D, Krausslic, HG, Dammacco F, Rice CM, Blum HE, Moradpour D (2000): Subcellular localization, stability, and trans-cleavage competence of the hepatitis C virus NS3-NS4A complex expressed in tetracyclineregulated cell lines. J. Virol. 74, 2293-2304. http://dx.doi. org/10.1128/JVI.74.5.2293-2304.2000

Yi M, Ma Y, Yates J, Lemon SM (2007): Compensatory mutations in E1, p7, NS2, and NS3 enhance yields of cell culture-infectious intergenotypic chimeric hepatitis $\mathrm{C}$ virus. J. Virol. 81, 629-638. http://dx.doi.org/10.1128/ JVI.01890-06

Yu GY, Lee KJ, Gao L, Lai MM (2006): Palmitoylation and polymerization of hepatitis C virus NS4B protein. J. Virol. 80, 6013-6023. http://dx.doi.org/10.1128/ JVI.00053-06 


\title{
Supplementary information
}

\section{Analysis of different HCV NS4B domains for the development of global consensus sequence}

\author{
H. KHAN, S. MURAD
}

Molecular Immunology Lab, Health Care Biotech Department, Atta-ur-Rahman School of Applied Biosciences, National University of Sciences and Technology, 44000 Islamabad, Pakistan

Received January 7, 2015; revised February 16, 2015; accepted August 12, 2015

Supplementary Table 1. List of Acc. Nos. of NS4B sequences included in the multiple sequence analysis

\begin{tabular}{|c|c|c|c|c|c|}
\hline 1a. NC004102 & 1a. AJ851228 & 1a. EU155345 & 1a. EU155272 & 1a. EU155273 & 1a. EU155274 \\
\hline 1a. EU482853 & 1a. EU155346 & 1a. EU155347 & 1a. EU155275 & 1a. EU482882 & 1a. EU155311 \\
\hline 1a. EU155348 & 1a. EU482854 & 1a. EU155349 & 1a. EU155312 & 1a. EU155313 & 1a. EU155314 \\
\hline 1a. EU482855 & 1a. EU482856 & 1a. EU529681 & 1a. EU482834 & 1a. EU155319 & 1a. EU155320 \\
\hline 1a. EU155350 & 1a. EU155351 & 1a. EU482872 & 1a. EU155321 & 1a. EU482835 & 1a. EU155322 \\
\hline 1a. EU155352 & 1a. EU482857 & 1a. EU155353 & 1a. EU155323 & 1a. EU155276 & 1a. EU155277 \\
\hline 1a. EU155354 & 1a. EU155355 & 1a. EU482858 & 1a. EU482843 & 1a. EU482844 & 1a. EU155278 \\
\hline 1a. EU482831 & 1a. EU155378 & 1a. EU482873 & 1a. EU482845 & 1a. EU482846 & 1a. EU155233 \\
\hline 1a. EU155379 & 1a. EU482832 & 1a. EU155380 & 1a. EU482861 & 1a. EU155236 & 1a. EU482862 \\
\hline 1a. D10749 & 1a. DQ889251 & 1a. DQ889252 & 1a. EU482863 & 1a. EU155237 & 1a. EU482864 \\
\hline 1a. DQ889253 & 1a. DQ889254 & 1a. DQ889255 & 1a. EU482865 & 1a. EU482866 & 1a. EU482867 \\
\hline 1a. DQ889256 & 1a. DQ889257 & 1a. DQ889258 & 1a. EU155238 & 1a. EU482847 & 1a. EU482836 \\
\hline 1a. DQ889259 & 1a. DQ889260 & 1a. DQ889261 & 1a. EU250017 & 1a. EU529677 & 1a. EU155239 \\
\hline 1a. DQ889262 & 1a. DQ889263 & 1a. DQ889264 & 1a. EU529678 & 1a. EU482887 & 1a. EU155240 \\
\hline 1a. DQ889265 & 1a. DQ889266 & 1a. DQ889267 & 1a. EU482868 & 1a. EU155282 & 1a. EU155283 \\
\hline 1a. DQ889268 & 1a. DQ889269 & 1a. DQ889270 & 1a. EU482869 & 1a. EU155241 & 1a. EU155242 \\
\hline 1a. DQ889271 & 1a. DQ889272 & 1a. DQ889273 & 1a. EU482870 & 1a. EU529679 & 1a. EU482871 \\
\hline 1a. DQ889274 & 1a. DQ889275 & 1a. DQ889276 & 1a. EU529680 & 1a. EU155243 & 1a. EU482848 \\
\hline 1a. DQ889277 & 1a. DQ889278 & 1a. DQ889279 & 1a .EU155244 & 1a. EU155245 & 1a. EU155246 \\
\hline 1a. EF032883 & 1a. EF032884 & 1a. EF032885 & 1a. EF032900 & 1a. EF032895 & 1a. EF032891 \\
\hline 1a. DQ889280 & 1a. DQ889281 & 1a. DQ889282 & 1a. EF032890 & 1a. EF032886 & 1a. DQ889301 \\
\hline 1a. DQ889283 & 1a. DQ889284 & 1a. DQ889285 & 1a. DQ889302 & 1a. DQ889303 & 1a. DQ889304 \\
\hline 1a. DQ889286 & 1a. DQ889287 & 1a. DQ889288 & 1a. DQ889305 & 1a. DQ889306 & 1a. AF011753 \\
\hline 1a. DQ889289 & 1a. DQ889290 & 1a. DQ889291 & 1a. EF621489 & 1a. AY615798 & 1a. DQ889307 \\
\hline 1a. DQ889292 & 1a. DQ889293 & 1a. DQ889294 & 1a. DQ889308 & 1a. DQ889309 & 1a. DQ889310 \\
\hline 1a. DQ889295 & 1a. DQ889296 & 1a. DQ889297 & 1a. DQ889311 & 1a. DQ889312 & 1a. DQ889313 \\
\hline 1a. DQ889298 & 1a. DQ889299 & 1a. DQ889300 & 1a. DQ889314 & 1a. DQ889315 & 1a. DQ889316 \\
\hline
\end{tabular}


Supplementary Table 1. (continued)

\begin{tabular}{|c|c|c|c|c|c|}
\hline 1a. EU155247 & 1a. EU260395 & 1a.EU155213 & 1a. DQ889317 & 1a. DQ889318 & 1a. DQ889319 \\
\hline 1a. EU155214 & 1a. EU155215 & 1a. EU260396 & 1a. DQ889320 & 1a. DQ889321 & 1a. AY695437 \\
\hline 1a. EU155216 & 1a. EU155338 & 1a. EU482837 & 1a. DQ430811 & 1a. EF407412 & 1a. EF407457 \\
\hline 1a. EU155339 & 1a. EU482889 & 1a. EU234064 & 1a. EF407432 & 1a. EF407428 & 1a. EF407437 \\
\hline 1a. EU155340 & 1a. EU482850 & 1a. EU482878 & 1a. EF407441 & 1a. EF407452 & 1a. EF407434 \\
\hline 1a. EU155341 & 1a. EU155342 & 1a. EU155343 & 1a. EF407449 & 1a. AY956468 & 1a. AY956464 \\
\hline 1a. EU155344 & 1a. EU482852 & 1a. EU155284 & 1a. AY956469 & 1a. EF407431 & 1a. EF407415 \\
\hline 1a. EU155285 & 1a. EU155286 & 1a. EU155287 & 1a. EF407416 & 1a. EF407456 & 1a. EF407453 \\
\hline 1a. EU155288 & 1a. EU155289 & 1a. EU155290 & 1a. EF407417 & 1a. EF407419 & 1a. EF407445 \\
\hline 1a. EU155291 & 1a. EU155292 & 1a. EU155293 & 1a. EF407413 & 1a. EF407435 & 1a. EF407411 \\
\hline 1a. EU155294 & 1a .EU482876 & 1a. EU155295 & 1a. EF407424 & 1a. AY956466 & 1a. AY956463 \\
\hline 1a. EU155296 & 1a. EU155297 & 1a. EU155298 & 1a. AY956465 & 1a. EF407422 & 1a. EF407448 \\
\hline 1a. EU155299 & 1a. EU155248 & 1a. EU155249 & 1a. EF407450 & 1a. EF407427 & 1a. EF407444 \\
\hline 1a. EU155309 & 1a. EU155310 & 1a. EU155250 & 1a. EF407454 & 1a. EF407425 & 1a. EF407440 \\
\hline 1a. EU155251 & 1a. EU482838 & 1a. EU155252 & 1a. EF407430 & 1a. EF407438 & 1a. EF407414 \\
\hline 1a. EU482884 & 1a. EU482840 & 1a. EU482841 & 1a. EF407418 & 1a. EF407443 & 1a. EF407420 \\
\hline 1a. EU155265 & 1a. EU482842 & 1a. EU529676 & 1a. EF407436 & 1a. EF407451 & 1a. EF407429 \\
\hline 1a. EU155266 & 1a. EU155267 & 1a. EU155268 & 1a. EF407433 & 1a. EF407455 & 1a. EF407439 \\
\hline 1a. EU155269 & 1a. EU155270 & 1a. EU155271 & 1a. EF407447 & 1a. EF407446 & 1a. EF407442 \\
\hline 1a. EF407423 & 1a. EF407421 & 1a. EF407426 & 1b. EU155258 & 1b. EU482879 & 1b. EU482880 \\
\hline 1a. AF290978 & 1a. AJ278830 & 1a. M32084 & 1b. EU155259 & 1b. EU155260 & 1b. EU155261 \\
\hline 1a. DQ838739 & 1a. AF511948 & 1a. AF511949 & 1b. EU155262 & 1b. EU482881 & 1b. EU155263 \\
\hline 1a. AF511950 & 1a. AF271632 & 1b. AJ000009 & 1b. EU155264 & 1b. EU155315 & 1b. EU155316 \\
\hline 1b. EU482859 & 1b. EU155356 & 1b. EU155357 & 1b. EU155317 & 1b. EU155318 & 1b. EU155279 \\
\hline 1b. EU155358 & 1b. EU155359 & 1b. EU155360 & 1b. EU155280 & 1b. EU155234 & 1b. EU482885 \\
\hline 1b. EU155361 & 1b. EU155362 & 1b. EU155363 & 1b. EU155235 & 1b. EU482886 & 1b. EU155281 \\
\hline 1b. EU482874 & 1b. EU155364 & 1b. EU155365 & 1b. EF032894 & 1b .EF407459 & 1b. EF407473 \\
\hline 1b. EU529682 & 1b. EU155366 & 1b. EU155367 & 1b. EF407481 & 1b. EF407467 & 1b. EF407458 \\
\hline 1b. EU155368 & 1b. EU155369 & 1b. EU155370 & 1b. EF407495 & 1b. EF407500 & 1b. EF407502 \\
\hline 1b. EU155371 & 1b. EU482860 & 1b. EU155372 & 1b. EF407491 & 1b. EF407474 & 1b. EF407475 \\
\hline 1b. EU155373 & 1b. EU155374 & 1b. EU155375 & 1b. EF407476 & 1b. EF407493 & 1b. EF407478 \\
\hline 1b. EU482875 & 1b. EU155376 & 1b. EU155377 & 1b. EF407480 & 1b. EF407468 & 1b. EF407496 \\
\hline 1b. AY587016 & 1b. EF638081 & 1b. D10934 & 1b. EF407498 & 1b. EF407466 & 1b. EF407469 \\
\hline 1b. AY460204 & 1b. L02836 & 1b. EU155381 & 1b. EF407486 & 1b. EF407472 & 1b. EF407479 \\
\hline 1b. EU482833 & 1b. EU155382 & 1b. AJ238799 & 1b. EF407483 & 1b. EF407470 & 1b. EF407497 \\
\hline 1b. AJ132997 & 1b. U45476 & 1b. AJ238800 & 1b. EF407461 & 1b. EF407471 & 1b. EF407463 \\
\hline 1b. AB154206 & 1b. AB109543 & 1b. M58335 & 1b. EF407484 & 1b. EF407503 & 1b. EF407465 \\
\hline 1b. D90208 & 1b. D50480 & 1b. D50481 & 1b. EF407462 & 1b. EF407482 & 1b. EF407487 \\
\hline 1b. D50484 & 1b. AB426117 & 1b. AF139594 & 1b. EF407490 & 1b. EF407499 & 1b. EF407489 \\
\hline 1b. D63857 & 1b. AB191333 & 1b. D16435 & 1b. EF407492 & 1b. EF407485 & 1b. EF407501 \\
\hline 1b. D14484 & 1b. X61596 & 1b. D11355 & 1b. EF407494 & 1b. EF407460 & 1b. EF407488 \\
\hline 1b. DQ244140 & 1b. DQ244141 & 1b. AF165046 & 1b. EF407477 & 1b. EF407464 & 1b. EF407504 \\
\hline 1b. AF165064 & 1b. AF165048 & 1b. AF165050 & 1b. AB016785 & 1b. D89815 & 1b. AY045702 \\
\hline 1b. AF165052 & 1b. AF165054 & 1b. AF165056 & 1b. AF333324 & 1b. D85516 & 1b. AF356827 \\
\hline 1b. AF165058 & 1b. AF165060 & 1b. AF165062 & 1b. AB049087 & 1b. AB049088 & 1b. AB049089 \\
\hline 1b. D89872 & 1b. U26687 & 1b. U01214 & 1b. AB049090 & 1b. AB049091 & 1b. AB049092 \\
\hline
\end{tabular}


Supplementary Table 1. (continued)

\begin{tabular}{|c|c|c|c|c|c|}
\hline 1b. AF176573 & 1b. AY044867 & 1b. AY587844 & 1b. AB049093 & 1b. AB049094 & 1b. AB049095 \\
\hline 1b. AF483269 & 1b. U89019 & 1b. M84754 & 1b. AB049096 & 1b. AB049097 & 1b. AB049098 \\
\hline 1b. EU155324 & 1b. EU155325 & 1b. EU482888 & 1b. АВ049099 & 1b. AB049100 & 1b. AB049101 \\
\hline 1b. EU155326 & 1b. EU155327 & 1b. EU234061 & 1b. D45172 & 1b. D30613 & 1b. .M96362 \\
\hline 1b. EU155328 & 1b. EU155329 & 1b. EU155330 & 1b. AB080299 & 1b. AF207752 & 1b. AF207753 \\
\hline 1b. EU155331 & 1b. EU155332 & 1b.EU155333 & 1b. AF207754 & 1b. AF207755 & 1b. AF207756 \\
\hline 1b. EU155334 & 1b. EU234062 & 1b. EU155335 & 1b. AF207757 & 1b. AF207758 & 1b. AF207759 \\
\hline 1b. EU482849 & 1b. EU155336 & 1b. EU155337 & 1b. AF207760 & 1b. AF207761 & 1b. AF207762 \\
\hline 1b. EU155217 & 1b. EU155218 & 1b. EU155219 & 1b. AF207763 & 1b. AF207764 & 1b. AF207765 \\
\hline 1b. EU155220 & 1b. EU155221 & 1b. EU155222 & 1b. AF207766 & 1b. AF207767 & 1b. AF207768 \\
\hline 1b. EU155223 & 1b..EU155224 & 1b. EU482883 & 1b. AF207769 & 1b. .AF207770 & 1b. AF207771 \\
\hline 1b. EU155225 & 1b. EU155226 & 1b. EU155227 & 1b. AF207772 & 1b. AF207773 & 1b. AF207774 \\
\hline 1b. EU155228 & 1b. EU155229 & 1b. EU155230 & 1b. AF208024 & 1b. AF054259 & 1b. AF313916 \\
\hline 1b. EU155231 & 1b. EU155232 & 1b. EU155300 & 1b/2k. AY587845 & 1c. D14853 & 1c. AY051292 \\
\hline 1b. EU482877 & 1b. EU155301 & 1b. EU155302 & 1c. AY651061 & 2/5. EF026073 & 2a. AY746460 \\
\hline 1b. EU155303 & 1b. EU155304 & 1b. EU155305 & 2a. AB047645 & 2a. .AF169003 & 2a. AF169004 \\
\hline 1b. EU155306 & 1b. EU155307 & 1b. EU155308 & 2a. 009823 & 2a. AF238481 & 2a. AF238482 \\
\hline 1b. EU155253 & 1b. EU155254 & 1b. EU155255 & 2a. AF238483 & 2a. AF238484 & 2a. AF238485 \\
\hline 1b. EU155256 & 1b. EU155257 & 1b. EU482839 & 2a. AF169002 & 2a. AF169005 & 2b. D10988 \\
\hline 2b. AY232731 & 2b. AY232749 & 2b. AY232733 & 2b. AY232741 & 2b. AY232743 & 2b. AY232745 \\
\hline 2b. AY232735 & 2b. AY232737 & 2b. AY232739 & 2b. AY232747 & 2b. DQ430815 & 2b. AB030907 \\
\hline 2b. AF238486 & 2b/1b. Q364460 & 2c. D50409 & 2i. DQ155561 & 2i/6p. DQ155560 & 2k. AB031663 \\
\hline 3a.YP.001491555.1 & 3a.AED87022.1 & 3a.ADR73013.1 & 3a.ACZ60107.1 & 3a.ACZ60106.1 & 3a.ACZ60105.1 \\
\hline 3a.ADI58378.1 & 3a.ACT79637.1 & 3a.AED02428.1 & 3a.ACZ60104.1 & 3a.ACZ60103.1 & 3a.ACZ60102.1 \\
\hline 3a.YP_001469631.1 & 3a.AGQ17416.1 & 3a.AGQ17415.1 & 3a.ADE10208.1 & 3a.AFH74072.1 & 3a.AFH74071.1 \\
\hline 3a.AGQ17413.1 & 3a.AGQ17412.1 & 3a.AHH29576.1 & 3a.AFH74070.1 & 3a.AFH74069.1 & 3a.AFH74068.1 \\
\hline 3a.BAM38518.1 & 3a.BAM38517.1 & 3a.AFA36246.1 & 3a.AFH74067.1 & 3a.AFH74066.1 & 3i.AFN53804.1 \\
\hline 3a.ACZ60118.1 & 3a.ACZ60117.1 & 3a.ACZ60114.1 & 3a.ACZ60109.1 & 3a.ACZ60108.1 & 3g.AFN53790.1 \\
\hline 3a.ACZ60113.1 & 3a.ACZ60112.1 & 3a.ACZ60111.1 & 3k.BAA09890.1 & 3i.AFN53792.1 & 3i.AFN53791.1 \\
\hline 3a.ACZ60110.1 & 4n.ACS29440.1 & 4o.ACS29439.1 & 4r.ACS29438.1 & 4k.ACS29437.1 & 4d.ACS29436.1 \\
\hline 4b.ACS29434.1 & 4q.ACS29433.1 & 4m.ACS29432.1 & 4a. DQ988077 & 4a. DQ418782 & 4a. DQ418783 \\
\hline 4g.ACS29431.1 & 4p.ACS29430.1 & 4v.AFN53795.1 & 4a. DQ418784 & 4a. DQ516084 & 4a. DQ418788 \\
\hline 4v.AFN53796.1 & 4. .DQ418785 & 4a. Y11604 & 4a. DQ418787 & 4a. .DQ418789 & 4d. DQ418786 \\
\hline 4a. DQ988078 & 4a. DQ988079 & 4a. DQ988073 & 4d. DQ516083 & 4f. EF589160 & 4f. EF589161 \\
\hline 4a. DQ988074 & 4a. DQ988075 & 4a. DQ988076 & 5a.YP_001469633.1 & 5a.AHH29581.1 & 5a.Y13184.1 \\
\hline 5a.CAA73640.1 & 5a.AAC61696.1 & 5a.AGS09785.1 & 5a.AGS09786.1 & 5a.AGS09783.1 & 5a.AGS09784.1 \\
\hline 5a.AGS09781.1 & 6. DQ278892 & 6a. AY859526 & 6a. DQ480514 & 6a. DQ480515 & 6a. DQ480518 \\
\hline 6a. DQ480513 & 6a. DQ480516 & 6a. DQ480523 & 6a. DQ480519 & 6a. DQ480520 & 6a. DQ480521 \\
\hline 6g. D63822 & 6h. D84265 & 6i. DQ835762 & 6a. DQ480522 & 6a. DQ480517 & 6a. DQ480524 \\
\hline 6i. DQ835770 & 6j. DQ835761 & 6j. DQ835769 & 6a. DQ480512 & 6a. Y12083 & 6b. NC_009827 \\
\hline 6k. DQ278893 & 6k. DQ278891 & 6k. D84264 & 6c. EF424629 & 6d. D84263 & 6e. DQ314805 \\
\hline 61. EF424628 & 6m. DQ835767 & 6m. DQ835765 & 6f. DQ835760 & 6f. DQ835764 & 6g. DQ314806 \\
\hline 6m. DQ835766 & 6m. DQ835763 & 6n. DQ278894 & 6n. DQ835768 & 6o. EF424627 & 6p. EF424626 \\
\hline 6t. EF632071 & 6q. EF424625 & 6t. .EF632069 & 6t. EF632070 & 7a. EF108306 & \\
\hline
\end{tabular}




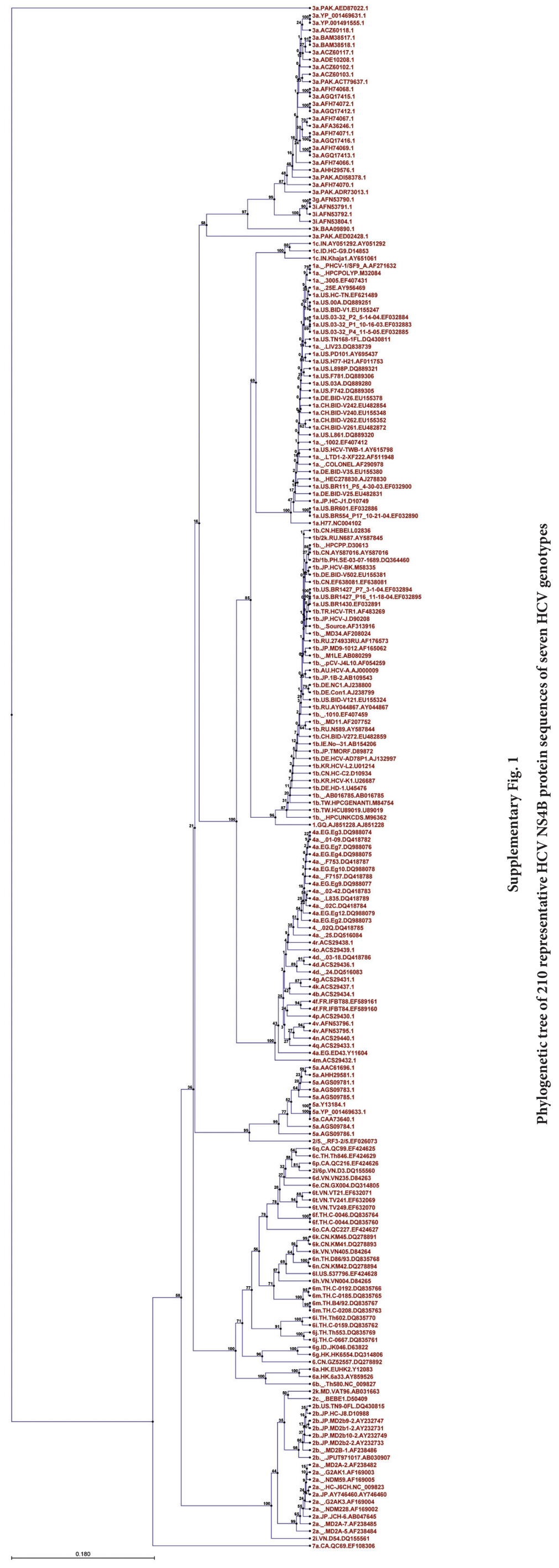

